\title{
ON STRONG DELTA-CONVEXITY AND HERMITE-HADAMARD TYPE INEQUALITIES FOR DELTA-CONVEX FUNCTIONS OF HIGHER ORDER
}

\author{
TERESA RAJBA
}

Abstract. In our previous paper [15], using $s$-convex stochastic ordering [4], we investigate Hermite-Hadamard-Fejér type inequalities in the case of higher order convex functions. In the present paper, our aim is to extend this investigation from convex to delta-convex functions of higher order [8]. We offer some useful tools for obtaining and proving of various forms of the Hermite-Hadamard-Fejér type inequalities for delta-convex functions of higher order, that generalizes results of Dragomir et al. [5]. These results are applied to derive some inequalities between quadrature operators. We define also and study strong delta-convexity of $n$-th order that generalizes strong $n$-convexity studied in [14] and [9].

Mathematics subject classification (2010): 26A51, 39B62.

Keywords and phrases: higher-order convexity, delta-convexity of higher order, control function, strong convexity, Hermite-Hadamard-Fejér type inequality.

\section{REFERENCES}

[1] M. BessenyeI, Hermite-Hadamard-type inequalities for generalized convex functions, J. Inequal. Pure Appl. Math., 9 (2008), 1-51.

[2] P. Billingsley, Probability and Measure, John Wiley \& Sons, New York, 1995.

[3] H. BRASS AND K. Petras, Quadrature theory. The theory of numerical integration on a compact interval, Mathematical Surveys and Monographs, 178, American Mathematical Society, Providence, RI, 2011.

[4] M. Denuit, C. LefÈvRe AND M. ShaKed, The s-convex orders among real random variables, with applications, Math. Inequal. Appl., 1 (1998), 585-613.

[5] S. S. Dragomir, C. E. M. Pearce, J. PeČarić, Means, g-Convex Dominated Functions and Hadamard-Type Inequalities, Tamsui Oxford Journal of Mathematical Sciences, 18 (2) (2002), 161173.

[6] S. S. Dragomir And C. E. M. Pearce, Selected Topics on Hermite-Hadamard Inequalities and Applications, RGMIA Monographs, Victoria University, 2000.

(Online: http://rgmia.vu.edu.au/monographs/).

[7] L. FEJÉR, Über die Fourierreihen, II, Math. Naturwiss. Anz. Ungar. Akad. Wiss., 24 (1906), 369-390.

[8] R. GER, Stability of polynomial mappings controlled by $n$-convex functionals, World Sci. Publ. Company (WSSIAA), 3 (1994) 255-268.

[9] R. Ger AND K. NiKODEM, Strongly convex functions of higher order, Nonlinear Anal., 74 (2011), 661-665.

[10] P. Hartman, On functions representable as difference of convex functions, Pacific J. Math., 9 (1959) 707-713.

[11] M. KucZMA, An Introduction to the Theory of Functional Equations and Inequalities, Prace Naukowe Uniwersytetu Śląskiego w Katowicach, vol. 489, Państwowe Wydawnictwo Naukowe - Uniwersytet Śląski, Warszawa, Kraków, Katowice, 1985.

[12] D. S. MitrinoviĆ And I. B. Lacković, Hermite and convexity, Aequationes Math., 28 (3) (1985), 229-232.

[13] J. E. Pečarić, F. Proschan, Y. L. Tong, Convex functions, Academic Press, Inc., 1992. 
[14] T. RAJBA, New integral representations of $n$th order convex functions, J. Math. Anal. Appl., 379 (2) (2011), 736-747.

[15] T. RAJBA, On the Ohlin lemma for Hermite-Hadamard-Fejér type inequalities, Math. Inequal. Appl. 17, 2 (2014) 557-571.

[16] A. Ralston, A First Course in Numerical Analysis, McGraw-Hill Book Company, New York, St. Louis, San Francisco, Toronto, London, Sydney, 1965.

[17] A. W. Roberts, D. E. Varberg, Convex Functions, Pure and Applied Mathematics, vol. 57, Academic Press, New York-London, 1973.

[18] H. L. Royden, Real analysis, Collier Macmillan, 1966.

[19] L. VeselÝ, L. ZAJIČEK, Delta-convex mappings between Banach spaces and applications, Dissertationes Math., 289, Polish Scientific Publishers, Warszawa, 1989.

[20] SZ. WAsSOWICZ, Inequalities between the quadrature operators and error bounds of quadrature rules, J. Inequal. Pure Appl. Math., 8 (2007), no. 2., Article 42, 8 pp.

[21] Sz. WA̧sowicz, On quadrature rules, inequalities and error bounds, J. Inequal. Pure Appl. Math., 9 (2008), no. 2, Article 36, 4 pp.

[22] Sz. WAssowICZ, On some extremalities in the approximate integration, Math. Inequal. Appl., 13 (2010), 165-174.

[23] E. W. Weisstein, Chebyshev Quadrature, From MathWorld - A Wolfram Web Resource. (Online: http://mathworld.wolfram.com/ChebyshevQuadrature.html).

[24] E. W. Weisstein, Legendre-Gauss Quadrature, From MathWorld - A Wolfram Web Resource. (Online: http://mathworld.wolfram.com/Legendre-GaussQuadrature.html).

[25] E. W. Weisstein, Lobatto Quadrature, From MathWorld - A Wolfram Web Resource. (Online: http://mathworld.wolfram.com/LobattoQuadrature.html).

[26] E. W. Weisstein, Simpson's Rule, From MathWorld - A Wolfram Web Resource. (Online: http://mathworld.wolfram.com/SimpsonsRule.html). 